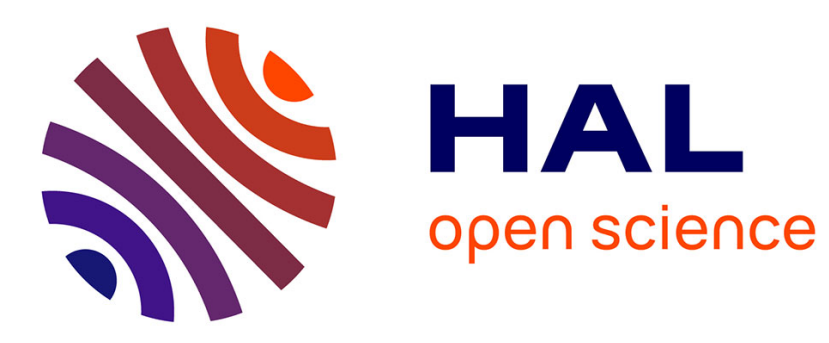

\title{
Synthesis and biological activities of aminopyrimidyl-indoles structurally related to meridianins
}

Rufine Akué-Gédu, Eric Debiton, Yoan Ferandin, Laurent Meijer, Michelle Prudhomme, Fabrice Anizon, Pascale Moreau

\section{To cite this version:}

Rufine Akué-Gédu, Eric Debiton, Yoan Ferandin, Laurent Meijer, Michelle Prudhomme, et al.. Synthesis and biological activities of aminopyrimidyl-indoles structurally related to meridianins. Bioorganic and Medicinal Chemistry, 2009, 17, pp.4420-4424. hal-00392799

\section{HAL Id: hal-00392799 \\ https://hal.science/hal-00392799}

Submitted on 9 Jun 2009

HAL is a multi-disciplinary open access archive for the deposit and dissemination of scientific research documents, whether they are published or not. The documents may come from teaching and research institutions in France or abroad, or from public or private research centers.
L'archive ouverte pluridisciplinaire HAL, est destinée au dépôt et à la diffusion de documents scientifiques de niveau recherche, publiés ou non, émanant des établissements d'enseignement et de recherche français ou étrangers, des laboratoires publics ou privés. 


\title{
Synthesis and biological activities of aminopyrimidyl-indoles structurally related to meridianins
}

Rufine Akue-Gedu ${ }^{\mathrm{a}, \mathrm{b}}$, Eric Debiton ${ }^{\mathrm{c}}$, Yoan Ferandin ${ }^{\mathrm{d}}$, Laurent Meijer ${ }^{\mathrm{d}}$, Michelle Prudhomme ${ }^{\mathrm{a}, \mathrm{b}}$, Fabrice Anizon $^{\mathrm{a}, \mathrm{b}}$, and Pascale Moreau ${ }^{\mathrm{a}, \mathrm{b}}$

${ }^{a}$ Clermont Université, Université Blaise Pascal, SEESIB, 24 avenue des Landais, F-63177 Aubière, France

${ }^{\mathrm{b}} \mathrm{CNRS}$, UMR 6504, F-63177 Aubière, France

${ }^{c}$ Clermont Université, Université d'Auvergne, Centre Jean Perrin, EA 4231, F-63005 Clermont-Ferrand, France

${ }^{\mathrm{d}}$ CNRS, Protein Phosphorylation \& Human Disease Group, Station Biologique, BP 74, 29682 Roscoff, France

\begin{abstract}
The synthesis of new meridianin derivatives substituted at the C-5 position of the 2-aminopyrimidine ring by various aryl groups and substituted or not by a methyl group on the indole nitrogen is described. These compounds were tested for their kinase inhibitory potencies toward five kinases (CDK5/p25, CK $1 \delta / \varepsilon$, GSK-3 $\alpha / \beta$, Dyrk1A and Erk2) as well as their in vitro antiproliferative activities toward a human fibroblast primary culture and two human solid cancer cell lines (MCF-7 and PA 1).
\end{abstract}

Keywords: Meridianins; Suzuki cross-coupling; Kinase inhibitors; Dyrk1A inhibitors

\section{Introduction}

Meridianins are marine alkaloids isolated and characterized from the south Atlantic tunicate Aplidium meridianum. ${ }^{1}$ Meridianins $A-G^{[1],[2] ~ a n d ~[3] ~}$ (Fig. 1), indole derivatives substituted in the C-3 position by a 2-aminopyrimidine ring, were successfully evaluated for their ability to inhibit various protein kinases ${ }^{3}$ and to display antitumour activity. ${ }^{4}$ The closely related meriolins, a family of 7 -azaindole synthetic meridianin analogues, which exhibit potent kinase inhibitory activities and antiproliferative properties were also described. ${ }^{[5]}$ and [6]

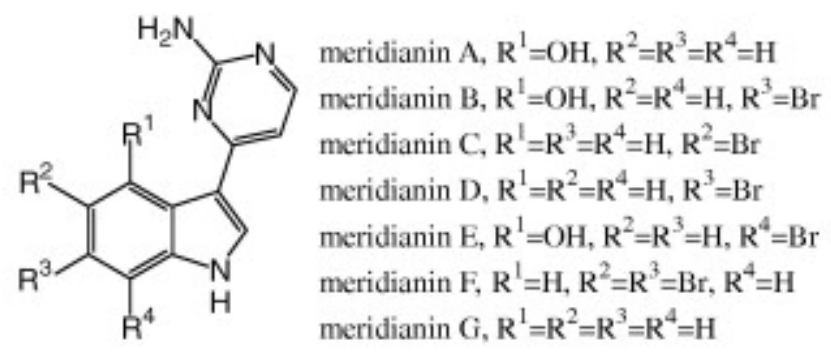
A $\mathrm{R}=\mathrm{H}, \mathrm{R}^{\prime}=\mathrm{Br}$
B $\mathrm{R}=\mathrm{H}, \mathrm{R}^{\prime}=$ phenyl
C $\mathrm{R}=\mathrm{H}, \mathrm{R}^{\prime}=4$-acetylphenyl
D $\mathrm{R}=\mathrm{H}, \mathrm{R}^{\prime}=4$-biphenyl
E $\mathrm{R}=\mathrm{H}, \mathrm{R}^{\prime}=4$-fluorophenyl
F $\mathrm{R}=\mathrm{H}, \mathrm{R}^{\prime}=$ 2,4-difluorophenyl
G $\mathrm{R}=\mathrm{H}, \mathrm{R}^{\prime}=4$-trifluoromethylphenyl
H R = H, R' = 4-trifluoromethoxyphenyl
I $\mathrm{R}=\mathrm{Me}, \mathrm{R}^{\prime}=\mathrm{H}$
J $\mathrm{R}=\mathrm{Me}, \mathrm{R}^{\prime}=\mathrm{Br}$
K $\mathrm{R}=\mathrm{Me}, \mathrm{R}^{\prime}=$ phenyl
L $\mathrm{R}=\mathrm{Me}, \mathrm{R}^{\prime}=4$-acetylphenyl
M R = Me, R'=4-biphenyl
N R=Me, R' = 4-fluorophenyl
O $\mathrm{R}=\mathrm{Me}, \mathrm{R}^{\prime}=2$,4-difluorophenyl
P $\mathrm{R}=\mathrm{Me}, \mathrm{R}^{\prime}=$ 4-trifluoromethylphenyl
Q R = Me, R' = 4-trifluoromethoxyphenyl

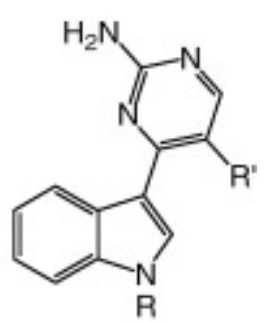

Figure 1. Meridianins A-G and derivatives $\mathbf{A}-\mathbf{Q}$ described by our group.

Since protein kinases are attractive targets for the discovery of biologically active compounds of potential therapeutic interest, our aim is to identify new ATP-competitive kinase inhibitors. Thus, we were recently interested in meridianin derivatives substituted at the C-5 position of the 2 -aminopyrimidine ring. In recent papers, we described the synthesis and biological activities of meridianin $\mathrm{G}$ derivatives, substituted or not on the indole nitrogen with a methyl group, and bearing various aryl moieties at the C-5 position of the pyrimidine ring (compounds $\mathbf{A}-\mathbf{H}$ and $\mathbf{J}-\mathbf{Q}$, Fig. 1).$^{[7] \text { and }}{ }^{[8]}$ It was shown that the substitution at this C-5 position and the methylation of the indole nitrogen were critical for either kinase inhibitory potencies 
or in vitro antiproliferative activities. The meridianin derivatives $\mathbf{A}-\mathbf{H}$ and $\mathbf{K}-\mathbf{Q}$ were tested toward a panel of 9 protein kinases (KDR, IGF-1R, c-Met, RET, c-Src, c-Abl, PKA, CDK2/cyclin A and HER-1) and their in vitro antiproliferative activities were examined toward a human fibroblast primary culture and two human solid cancer cell lines (MCF-7 and PA 1). Despite weak kinase inhibitory potencies, compounds $\mathbf{G}, \mathbf{H}, \mathbf{L}$ and $\mathbf{M}$ exhibited high in vitro antiproliferative activities toward PA 1 cells. It was found that these compounds do not interfere with PA 1 cell-cycle and may be considered as direct cytolysis or apoptosis inducers. Considering these first biological results, it was of interest to pursue the study with new aryl substituents on the pyrimidine ring in order to identify the substitution pattern providing the highest biological potencies.

In this paper, we described the synthesis of new meridianin analogues diversely substituted at the C-5 position of the pyrimidine ring and methylated or not on the indole nitrogen. Moreover these compounds were tested for their kinase inhibitory potencies toward five kinases (CDK5/p25, CK $1 \delta / \varepsilon$, GSK $3 \alpha / \beta$, Dyrk1A and Erk2) as well as their in vitro antiproliferative activities toward a human fibroblast primary culture and two human solid cancer cell lines (MCF-7 and PA 1).

\section{Chemistry}

Compounds 1-9 were synthesized from brominated derivatives $\mathbf{A}$ or $\mathbf{J}$, methylated or not on the indole nitrogen. A and $\mathbf{J}$ were prepared according to previously described procedure (Scheme 1). ${ }^{7}$ Suzuki crosscoupling was performed using various commercially available arylboronic acids, tetrakis(triphenylphosphine)palladium( 0 ) and sodium carbonate in a $\mathrm{H}_{2} \mathrm{O} / \mathrm{EtOH} /$ toluene medium. Compounds 1-9 were obtained in 31-60\% isolated yields. Due to purification difficulties, the nonmethylated analogues of compounds 7-9 could not be isolated as pure compounds.

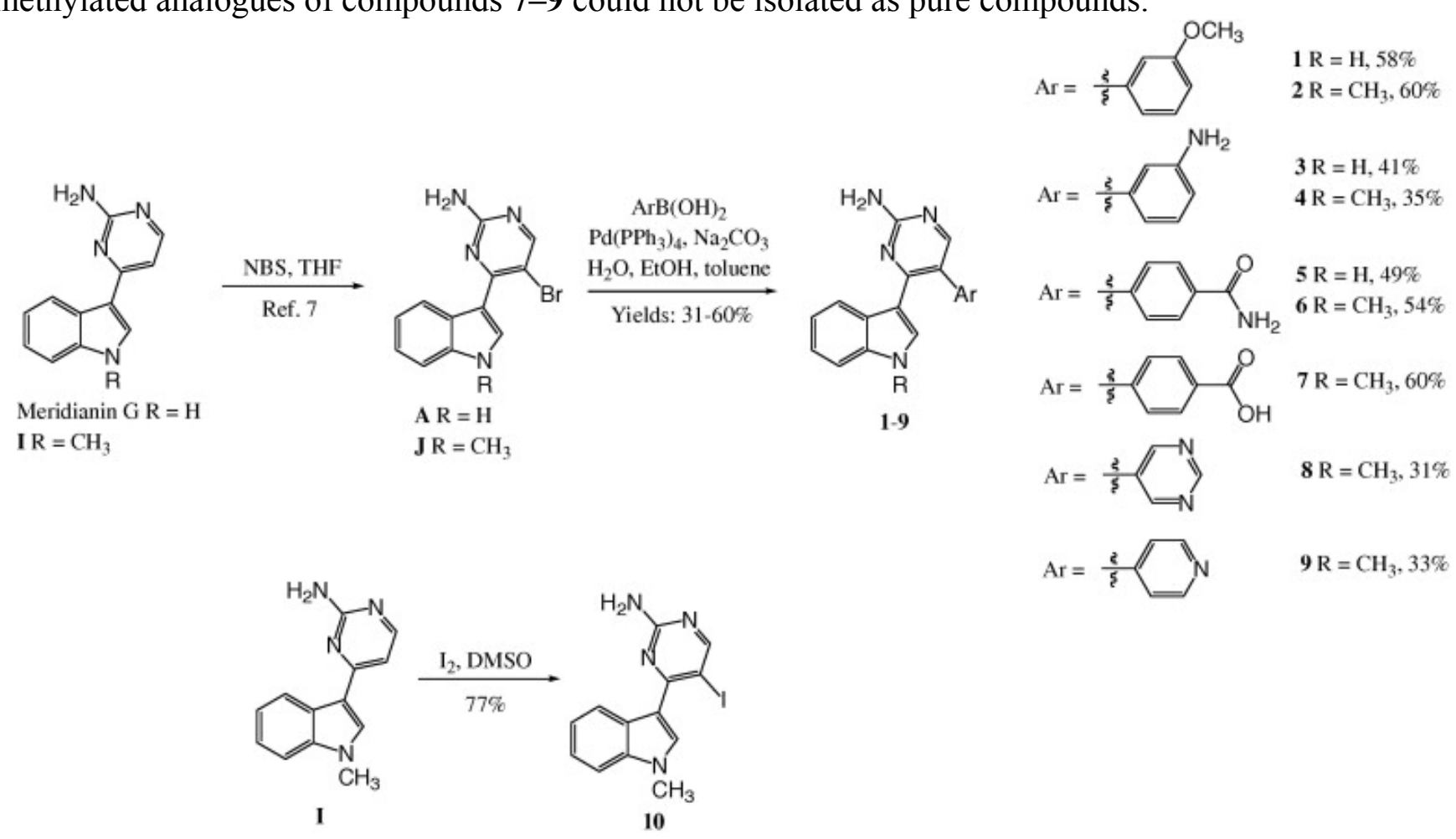

Scheme 1. Synthesis of compounds 1-10.

Results were similar using compounds $\mathbf{A}$ or $\mathbf{J}$ (compare $\mathbf{1}$ and 2, $\mathbf{3}$ and 4, $\mathbf{5}$ and $\mathbf{6}$ ), and yields dropped using aryl boronic acid containing a basic nitrogen atom.

Moreover, in order to introduce various amines (aryl or alkylamines) by Buchwald type coupling, in the C-5 position of the pyrimidine ring, compound $\mathbf{1 0}$, the iodo analogue of $\mathbf{J}$, was prepared in $77 \%$ yield from methylated meridianin $\mathrm{G}$ (compound I), using iodine in DMSO. 9

Unfortunately, we never manage to substitute this position by an amine derivative from compound $\mathbf{1 0}$ or its brominated analogue $\mathbf{J}$.

In summary, the method described here allowed the introduction, in the pyrimidine C-5 position, of heteroaryl moieties or phenyl groups bearing both electron donor or electron acceptor substituents in para or meta positions. 


\section{Results and discussion}

In vitro antiproliferative activities of compounds 1-10 were evaluated toward a human fibroblasts primary culture and two human solid cancer cell lines (MCF-7 and PA 1). The antiproliferative effect $\left(\mathrm{IC}_{50}\right)$ of the tested drug was assessed by the resazurin reduction test. ${ }^{10}$ Under the conditions used, none of the newly synthesised compounds showed any significant cytotoxic activity $\left(\mathrm{IC}_{50}<50 \mu \mathrm{M}\right)$ on fibroblasts or MCF-7 cell lines. Only mild activities $\left(\mathrm{IC}_{50}\right.$ around $\left.30 \mu \mathrm{M}\right)$ were observed on PA 1 cells with compounds 1, 2, 5 and $\mathbf{1 0}$ (Table 1). These results indicate that the substituents introduced at the C-5' position are detrimental to the cytotoxic effect of these series on PA 1 cells.

Table 1.

Inhibitory potencies toward five protein kinases $\left(\mathrm{IC}_{50}\right.$ in $\left.\mu \mathrm{M}\right)$ and antiproliferative activity of compounds $\mathbf{G}, \mathbf{H}, \mathbf{L}, \mathbf{M}$ and $\mathbf{1 - 1 0}\left(\mathrm{IC}_{50}\right.$ in $\left.\mu \mathrm{M}\right)$

\begin{tabular}{|c|c|c|c|c|c|c|}
\hline \multirow[t]{2}{*}{ Combounds } & \multicolumn{5}{|c|}{ Kinase inhibition- $-\mathrm{IC}_{50}$ in $\mathrm{uM}$} & \multirow[t]{2}{*}{ Antiproliferative activitv toward } \\
\hline & CDK5/p25 & CK1 $\delta / \varepsilon$ & GSK3 $\boldsymbol{\alpha} / \boldsymbol{\beta}$ & Dvrk1A & Erk2 & \\
\hline $\mathbf{G}$ & 11.0 & 8.3 & 1.9 & 2.3 & 0.58 & 0.09 \\
\hline $\mathbf{H}$ & $>10$ & $>10$ & 3.0 & 2.9 & 3.2 & 0.08 \\
\hline $\mathbf{L}$ & $>10$ & $>10$ & 1.4 & 5.0 & $>10$ & 0.05 \\
\hline $\mathbf{M}$ & $>10$ & $>10$ & $>10$ & $>10$ & $>10$ & 0.08 \\
\hline 1 & $>10$ & 0.49 & 3.0 & 0.60 & 3.10 & 28 \\
\hline 2 & $>10$ & 2.2 & 3.8 & 3.1 & $>10$ & 34 \\
\hline 3 & $>10$ & 3.3 & 6.0 & 0.64 & 6.9 & $>50$ \\
\hline 4 & $>10$ & 3.1 & 5.0 & 1.9 & $>10$ & $>50$ \\
\hline 5 & $>10$ & 3.4 & 2.0 & 0.58 & 1.90 & 38 \\
\hline 6 & $>10$ & 4.1 & 0.63 & 2.0 & 7.00 & $>50$ \\
\hline 7 & $>10$ & $>10$ & $>10$ & 0.9 & $>10$ & $>50$ \\
\hline 8 & $>10$ & 12.0 & 6.1 & 3.2 & $>10$ & $>50$ \\
\hline 9 & 5.3 & 4.2 & 1.2 & 2.4 & 1.30 & $>50$ \\
\hline 10 & 2.6 & 1.1 & 4.2 & 0.4 & 4.0 & 31 \\
\hline
\end{tabular}

The kinase inhibitory potencies of compounds 1-10 and that of previously synthesised derivatives $\mathbf{G}, \mathbf{H}$, $\mathbf{L}$ and $\mathbf{M}$ which have shown interesting antiproliferative activities toward PA1 cells were evaluated as $\mathrm{IC}_{50}$ values toward five protein kinases (CDK5/p25, CK1 $\delta / \varepsilon, \mathrm{GSK}-3 \alpha / \beta$, Dyrk1A and Erk2).

Only two compounds were mildly active toward CDK5/p25 with $\mathrm{IC}_{50}$ values in the micromolar range. These derivatives are both methylated on the indole nitrogen and substituted either by a pyridyl group (compound $9, \mathrm{IC}_{50}=5.3 \mu \mathrm{M}$ ) or by an iodine atom (compound $10, \mathrm{IC}_{50}=2.6 \mu \mathrm{M}$ ) in the $\mathrm{C}-5$ position of the aminopyrimidine moiety.

Considering the other kinases, the best inhibitory potencies were sub-micromolar inhibition of CK $1 \delta / \varepsilon$ (compound 1, $\mathrm{IC}_{50}=0.49 \mu \mathrm{M}$ ), GSK-3 $\alpha / \beta$ (compound 6, $\mathrm{IC}_{50}=0.63 \mu \mathrm{M}$ ), Dyrk1A (compounds 1, $\left.\mathrm{IC}_{50}=0.6 \mu \mathrm{M} ; 3, \mathrm{IC}_{50}=0.64 \mu \mathrm{M} ; \mathbf{5}, \mathrm{IC}_{50}=0.58 \mu \mathrm{M} ; 7, \mathrm{IC}_{50}=0.9 \mu \mathrm{M} ; \mathbf{1 0}, \mathrm{IC}_{50}=0.4 \mu \mathrm{M}\right)$ and Erk2 (compound $\mathbf{G}, \mathrm{IC}_{50}=0.58 \mu \mathrm{M}$ ). Regarding these results, this series of compounds could be particularly interesting in the development of new inhibitors of Dyrk1A kinase. Comparison of the activity of nonmethylated compounds $(\mathbf{1}, \mathbf{3}, \mathbf{5})$ toward Dyrk1 A with that of their $\mathrm{N}-\mathrm{CH}_{3}$ analogues $(\mathbf{2}, \mathbf{4}, \mathbf{6})$ indicate that the non-methylated analogues are more active than their methylated counterparts. Nevertheless, two methylated derivatives bearing either a $p$-carboxyphenyl moiety (7) or an iodine atom (10) at the C-5 position of the pyrimidine ring exhibited Dyrk1A sub-micromolar inhibition as well. 


\section{Conclusion}

Encouraging preliminary biological evaluation of diversely substituted meridianins led us to synthesize new derivatives. Suzuki cross-coupling could be applied to compounds A or $\mathbf{J}$, for coupling with either aromatic boronic acids bearing both electron donor and electron acceptor substituents or heteroaromatic boronic acids.

Surprisingly, the cytotoxic activity of newly synthesised derivatives $\mathbf{1 - 1 0}$ toward PA 1 cells is significantly smaller than that of the previously described compounds $\mathbf{G}, \mathbf{H}, \mathbf{L}, \mathbf{M}$. This could be due to a lower cellular penetration of compounds 1-10 or lower interaction, due to the modification of the aryl moiety, with the cellular target(s) involved in their antiproliferative activity.

Compounds G, 1, 3, 5-7 and $\mathbf{1 0}$ have shown sub-micromolar inhibition potencies toward some of the kinases tested. The interesting inhibition of Dyrk1A kinase, which is known to be involved in Alzheimer's disease ${ }^{11}$ and Down syndrome, ${ }^{12}$ leads us to expand our effort in the synthesis of diversely substituted meridianin derivatives as new potential inhibitors of this kinase.

\section{Experimental}

\subsection{In vitro Kinase inhibition assays}

\subsubsection{Buffers}

Buffer A: $10 \mathrm{mM} \mathrm{MgCl} 2,1 \mathrm{mM}$ EGTA, $1 \mathrm{mM}$ DTT, $25 \mathrm{mM}$ Tris-HCl pH 7.5, $50 \mu \mathrm{g}$ heparin/ml.

Buffer $C$ : $60 \mathrm{mM} \beta$-glycerophosphate, $15 \mathrm{mM}$ p-nitrophenylphosphate, $25 \mathrm{mM}$ Mops (pH 7.2), $5 \mathrm{mM}$ EGTA, $15 \mathrm{mM} \mathrm{MgCl}_{2}, 1 \mathrm{mM}$ DTT, $1 \mathrm{mM}$ sodium vanadate, $1 \mathrm{mM}$ phenylphosphate.

\subsubsection{Kinase preparations and assays}

Kinase activities were assayed in Buffer $\mathrm{A}$ or $\mathrm{C}$, at $30^{\circ} \mathrm{C}$, at a final ATP concentration of $15 \mu \mathrm{M}$. Blank values were subtracted and activities expressed in \% of the maximal activity, i.e. in the absence of inhibitors. Controls were performed with appropriate dilutions of DMSO.

$C D K 5 / p 25$ (human, recombinant) was prepared as previously described. ${ }^{[13]}$ and ${ }^{[14]}$ Its kinase activity was assayed in buffer $\mathrm{C}$, with $1 \mathrm{mg}$ histone $\mathrm{H} 1 / \mathrm{ml}$, in the presence of $15 \mu \mathrm{M}\left[\gamma^{33} \mathrm{P}\right]$ ATP $(3,000 \mathrm{Ci} / \mathrm{mmol}$; $10 \mathrm{mCi} / \mathrm{ml}$ ) in a final volume of $30 \mu \mathrm{l}$. After $30 \mathrm{~min}$ incubation at $30^{\circ} \mathrm{C}, 25 \mu \mathrm{l}$ aliquots of supernatant were spotted onto $2.5 \times 3 \mathrm{~cm}$ pieces of Whatman P81 phosphocellulose paper, and, $20 \mathrm{~s}$ later, the filters were washed five times (for at least $5 \mathrm{~min}$ each time) in a solution of $10 \mathrm{ml}$ phosphoric acid/liter of water. The wet filters were counted in the presence of $1 \mathrm{ml}$ ACS (Amersham) scintillation fluid.

GSK-3 $\alpha \beta$ (porcine brain, native) was assayed, as described for CDK5/p25 but in Buffer A and using a GSK-3 specific substrate (GS-1: YRRAAVPPSPSLSRHSSPHQSpEDEEE) (pS stands for phosphorylated serine). ${ }^{15}$ GS-1 was synthesized by Millegen (Labege, France).

$C K 1 \delta / \varepsilon$ (porcine brain, native) was assayed as described for CDK5/p25 but using the CK1-specific peptide substrate RRKHAAIGpSAYSITA, ${ }^{16}$ obtained from Millegen (Labege, France).

Erk2 (rat, recombinant) was assayed as described for CDK5/p25 but using the specific substrate Ets1 (amino acids 1-138) in buffer A.

DYRK1A (rat, recombinant, expressed in Escherichia coli as a GST fusion protein) was purified by affinity chromatography on glutathione-agarose and assayed as described for CDK5/p25 using myelin basic protein $(1 \mathrm{mg} / \mathrm{ml})$ as a substrate.

\subsection{In vitro antiproliferative assays}

\subsubsection{Cell cultures}

Stock cell cultures were maintained as monolayers in $75 \mathrm{~cm}^{2}$ culture flasks in Glutamax Eagle's minimum essential medium (MEM) with Earle's salts supplemented with $10 \%$ fetal calf serum, $5 \mathrm{ml}$ $100 \mathrm{mmol} / 1$ sodium pyruvate, $5 \mathrm{ml}$ of $100 \times$ non-essential amino acids and $2 \mathrm{mg}$ gentamicin base. Cells were grown at $37^{\circ} \mathrm{C}$ in a humidified incubator under an atmosphere containing $5 \% \mathrm{CO}_{2}$.

\subsubsection{Survival assays}

Cells were plated at a density of $5 \times 10^{3}$ cells in $150 \mu$ culture medium in each well of 96-well microplates and were allowed to adhere for $16 \mathrm{~h}$ before treatment with tested drug. A stock solution $20 \mathrm{mmol} / \mathrm{l}$ of each tested drug was prepared in DMSO and kept at $-20^{\circ} \mathrm{C}$ until use. Then $50 \mu \mathrm{l}$ of each tested solution were added to the cultures. A $48 \mathrm{~h}$ continuous drug exposure protocol was used. The antiproliferative effect of the tested drug was assessed by the resazurin reduction test. 


\subsubsection{Resazurin reduction test}

Plates were rinsed with $200 \mu \mathrm{PBS}$ at $37^{\circ} \mathrm{C}$ and emptied by overturning on absorbent toweling. Then $150 \mu \mathrm{l}$ of a $25 \mu \mathrm{g} / \mathrm{ml}$ solution of resazurin in MEM without phenol red was added to each well. Plates were incubated for $1 \mathrm{~h}$ at $37^{\circ} \mathrm{C}$ in a humidified atmosphere containing $5 \% \mathrm{CO}_{2}$. Fluorescence was then measured on an automated 96-well plate reader (Fluoroscan Ascent FL, Labsystem) using an excitation wavelength of $530 \mathrm{~nm}$ and an emission wavelength of $590 \mathrm{~nm}$. Under the conditions used, fluorescence was proportional to the number of living cells in the well. The $\mathrm{IC}_{50}$, defined as the drug concentration required to inhibit cell proliferation by $50 \%$, was calculated from the curve of concentration-dependent survival percentage, defined as fluorescence in experimental wells compared with fluorescence in control wells, after subtraction of the blank values.

\subsection{Chemistry}

IR spectra were recorded on a Perkin-Elmer Paragon 500 spectrometer $\bar{v}_{\text {maxin }} \mathrm{cm}^{-1}$ ). NMR spectra were performed on a Bruker AVANCE $400\left({ }^{1} \mathrm{H}: 400 \mathrm{MHz},{ }^{13} \mathrm{C}: 100 \mathrm{MHz}\right)$, chemical shifts $\delta$ are noted in ppm and the following abbreviations are used: singlet $(\mathrm{s})$, doublet $(\mathrm{d})$, triplet $(\mathrm{t})$, doublet of doublet $(\mathrm{dd})$, doublet of doublet of doublet (ddd), doublet of triplet (dt), multiplet (m), broad signal (br s). Mass spectra $($ ESI +$)$ were determined on a high resolution Waters Micro Q-Toff apparatus. Chromatographic purifications were performed on flash silica gel Geduran SI 60 (Merck) 0.040-0.063 mm column chromatography. TLC were performed on fluorescent silica gel plates (60 F254 from Merck).

\subsubsection{General procedure for the preparation of compounds 1-9}

A solution of $\mathrm{Na}_{2} \mathrm{CO}_{3}$ (2.5 equiv) in $\mathrm{H}_{2} \mathrm{O}(2 \mathrm{ml} / \mathrm{mmol})$ was added to a suspension of bromide $\mathbf{A}$ ( $288 \mathrm{mg}$, $1.0 \mathrm{mmol}$ ) or $\mathbf{J}(302 \mathrm{mg}, 1.0 \mathrm{mmol})$, the required boronic acid (1.1 equiv), $\mathrm{Pd}\left(\mathrm{PPh}_{3}\right)_{4}(57.8 \mathrm{mg}, 5 \mathrm{~mol} \%)$ in EtOH $(3 \mathrm{ml} / \mathrm{mmol})$ and toluene $(3 \mathrm{ml} / \mathrm{mmol})$. The reaction mixture was refluxed overnight under argon and the cooled resulting mixture was evaporated onto silica gel. Purification as indicated provided the desired compounds.

\subsubsection{4-(1H-Indol-3-yl)-5-(3-methoxyphenyl)pyrimidin-2-amine (1)}

Purification of the residue by flash chromatography (cyclohexane/EtOAc 7:3) provided 1 (185 mg, 58\%) as a yellow powder.

Mp 98-100 ${ }^{\circ} \mathrm{C}$; HRMS (ESI+) calcd for $\mathrm{C}_{19} \mathrm{H}_{17} \mathrm{~N}_{4} \mathrm{O}(\mathrm{M}+\mathrm{H})^{+}$317.1402, found 317.1385; IR (KBr): 3650-3150, $1605 \mathrm{~cm}^{-1}$; ${ }^{1} \mathrm{H}$ NMR (400 MHz, DMSO-d $): 3.71\left(3 \mathrm{H}, \mathrm{s}, \mathrm{CH}_{3}\right), 6.52-6.58\left(2 \mathrm{H}, \mathrm{br} \mathrm{s}, \mathrm{NH}_{2}\right)$, $6.69(1 \mathrm{H}, \mathrm{d}, J=3.0 \mathrm{~Hz}), 6.83-6.86(2 \mathrm{H}, \mathrm{m}), 6.91-6.95(1 \mathrm{H}, \mathrm{m}), 7.06\left(1 \mathrm{H}, \mathrm{ddd}, J_{1}=8.0 \mathrm{~Hz}, J_{2}=7.0 \mathrm{~Hz}\right.$; $\left.J_{3}=1.0 \mathrm{~Hz}\right), 7.12\left(1 \mathrm{H}, \mathrm{ddd}, J_{1}=8.0 \mathrm{~Hz}, J_{2}=7.0 \mathrm{~Hz}, J_{3}=1.0 \mathrm{~Hz}\right), 7.31(1 \mathrm{H}, \mathrm{t}, J=8.0 \mathrm{~Hz}), 7.34(1 \mathrm{H}, \mathrm{d}$, $J=8.0 \mathrm{~Hz}), 8.00(1 \mathrm{H}, \mathrm{s}), 8.46(1 \mathrm{H}, \mathrm{d}, J=8.0 \mathrm{~Hz}), 11.23-11.33(1 \mathrm{H}, \mathrm{br}, \mathrm{NH}) ;{ }^{13} \mathrm{C} \mathrm{NMR}(100 \mathrm{MHz}$, DMSO- $\left.d_{6}\right): 55.0\left(\mathrm{OCH}_{3}\right), 111.4,112.6,114.9,120.0,121.8,121.9,122.9,128.9,129.8,157.8(\mathrm{CH})$, $112.7,120.9,126.2,135.8,140.3,159.5,159.8,162.5(\mathrm{C})$.

\subsubsection{4-(1-Methyl-1H-indol-3-yl)-5-(3-methoxyphenyl)pyrimidin-2-amine (2)}

Purification of the residue by flash chromatography (cyclohexane/EtOAc 7:3) provided 2 (198 mg, 60\%) as a yellow powder.

$\mathrm{Mp}=82-84^{\circ} \mathrm{C}$; HRMS (ESI+) calcd for $\mathrm{C}_{20} \mathrm{H}_{19} \mathrm{~N}_{4} \mathrm{O}(\mathrm{M}+\mathrm{H})^{+}$331.1559, found 331.1546; IR (KBr): 3650-3150, $1604 \mathrm{~cm}^{-1}$; ${ }^{1} \mathrm{H}$ NMR $\left(400 \mathrm{MHz}, \mathrm{DMSO}-d_{6}\right): 3.61\left(3 \mathrm{H}, \mathrm{s}, \mathrm{NCH}_{3}\right), 3.72\left(3 \mathrm{H}, \mathrm{s}, \mathrm{OCH}_{3}\right)$, 6.56-6.59 $\left(2 \mathrm{H}\right.$, br s, $\left.\mathrm{NH}_{2}\right), 6.73(1 \mathrm{H}, \mathrm{s}), 6.83\left(1 \mathrm{H}, \mathrm{ddd}, J_{1}=7.5 \mathrm{~Hz}, J_{2}=1.5 \mathrm{~Hz}, J_{3}=1.0 \mathrm{~Hz}\right), 6.86(1 \mathrm{H}$, $\left.\mathrm{dd}, J_{1}=2.5 \mathrm{~Hz}, J_{2}=1.5 \mathrm{~Hz}\right), 6.93\left(1 \mathrm{H}, \mathrm{ddd}, J_{1}=8.5 \mathrm{~Hz}, J_{2}=2.5 \mathrm{~Hz}, J_{3}=1.0 \mathrm{~Hz}\right), 7.08(1 \mathrm{H}$, ddd, $\left.J_{1}=8.0 \mathrm{~Hz}, J_{2}=7.0 \mathrm{~Hz}, J_{3}=1.0 \mathrm{~Hz}\right), 7.18\left(1 \mathrm{H}, \mathrm{ddd}, J_{1}=8.0 \mathrm{~Hz}, J_{2}=7.0 \mathrm{~Hz}, J_{3}=1.0 \mathrm{~Hz}\right), 7.29(1 \mathrm{H}, \mathrm{t}$, $J=8.0 \mathrm{~Hz}), 7.40(1 \mathrm{H}, \mathrm{d}, J=8.0 \mathrm{~Hz}), 8.03(1 \mathrm{H}, \mathrm{s}), 8.35(1 \mathrm{H}, \mathrm{d}, J=8.0 \mathrm{~Hz}),{ }^{13} \mathrm{C}$ NMR $(100 \mathrm{MHz}, \mathrm{DMSO}-$ $\left.d_{6}\right): 32.8\left(\mathrm{CH}_{3}\right), 55.0\left(\mathrm{OCH}_{3}\right), 109.8,112.7,114.8,120.2,121.7,121.9,122.8,129.7,132.6,158.1(\mathrm{CH})$, $112.0,120.8,126.5,136.4,140.0,159.4,159.5,162.4(\mathrm{C})$.

\subsubsection{4-(1H-Indol-3-yl)-5-(3-aminophenyl)pyrimidin-2-amine (3)}

Purification of the residue by flash chromatography (cyclohexane/EtOAc 4:6) provided 3 (125 mg, 41\%) as a yellow powder. 
$\mathrm{Mp}>250^{\circ} \mathrm{C}$, decomposition; HRMS (ESI+) calcd for $\mathrm{C}_{18} \mathrm{H}_{16} \mathrm{~N}_{5}(\mathrm{M}+\mathrm{H})^{+}$302.1406, found 302.1397; IR (KBr): $3550-3100,1614 \mathrm{~cm}^{-1}$; ${ }^{1} \mathrm{H}$ NMR $\left(400 \mathrm{MHz}, \mathrm{DMSO}-d_{6}\right): 5.09-5.16\left(2 \mathrm{H}\right.$, br s, $\left.\mathrm{NH}_{2}\right), 6.42(1 \mathrm{H}$, ddd, $\left.J_{1}=7.5 \mathrm{~Hz}, J_{2}=1.5 \mathrm{~Hz}, J_{3}=1.0 \mathrm{~Hz}\right), 6.45-6.51\left(3 \mathrm{H}\right.$, br s, $\left.1 \mathrm{CH}+\mathrm{NH}_{2}\right), 6.57\left(1 \mathrm{H}, \mathrm{ddd}, J_{1}=8.0 \mathrm{~Hz}\right.$, $\left.J_{2}=2.5 \mathrm{~Hz}, J_{3}=1.0 \mathrm{~Hz}\right), 6.75(1 \mathrm{H}, \mathrm{d}, J=3.0 \mathrm{~Hz}), 7.05-7.16(3 \mathrm{H}, \mathrm{m}), 7.34(1 \mathrm{H}, \mathrm{d}, J=8.0 \mathrm{~Hz}), 7.91(1 \mathrm{H}$, s), $8.62(1 \mathrm{H}, \mathrm{d}, J=8.0 \mathrm{~Hz}), 11.25-11.35(1 \mathrm{H}, \mathrm{br} \mathrm{s}, \mathrm{NH}) ;{ }^{13} \mathrm{C}$ NMR $\left(100 \mathrm{MHz}, \mathrm{DMSO}-d_{6}\right): 111.3,112.9$, $114.7,116.8,120.0,121.8,123.2,129.2,129.3,157.4(\mathrm{CH}), 112.7,121.7,126.4,135.8,139.6,149.1$, $159.8,162.3(\mathrm{C})$.

\subsubsection{4-(1-Methyl-1H-indol-3-yl)-5-(3-aminophenyl)pyrimidin-2-amine (4)}

Purification of the residue by flash chromatography (cyclohexane/EtOAc 4:6) provided 4 (110 mg, 35\%) as a yellow powder.

$\mathrm{Mp}=183-185^{\circ} \mathrm{C}$; HRMS $(\mathrm{ESI}+)$ calcd for $\mathrm{C}_{19} \mathrm{H}_{18} \mathrm{~N}_{5}(\mathrm{M}+\mathrm{H})^{+}$316.1562, found 316.1548; IR $(\mathrm{KBr})$ : 3600-3100, 1651, $1602 \mathrm{~cm}^{-1}$; ${ }^{1} \mathrm{H}$ NMR $\left(400 \mathrm{MHz}, \mathrm{DMSO}-d_{6}\right): 3.60\left(3 \mathrm{H}, \mathrm{s}, \mathrm{CH}_{3}\right), 5.08-5.16(2 \mathrm{H}$, br s, $\left.\mathrm{NH}_{2}\right), 6.41(1 \mathrm{H}, \mathrm{d}, J=7.5 \mathrm{~Hz}), 6.48(1 \mathrm{H}, \mathrm{t}, J=1.5 \mathrm{~Hz}), 6.48-6.52\left(2 \mathrm{H}\right.$, br s, NH $\left.\mathrm{N}_{2}\right), 6.58(1 \mathrm{H}, \mathrm{dd}$, $\left.J_{1}=8.0 \mathrm{~Hz}, J_{2}=1.5 \mathrm{~Hz}\right), 6.73(1 \mathrm{H}, \mathrm{s}), 7.06(1 \mathrm{H}, \mathrm{t}, J=7.5 \mathrm{~Hz}), 7.11\left(1 \mathrm{H}\right.$, ddd, $J_{1}=8.0 \mathrm{~Hz}, J_{2}=7.0 \mathrm{~Hz}$, $\left.J_{3}=1.0 \mathrm{~Hz}\right), 7.19\left(1 \mathrm{H}, \mathrm{ddd}, J_{1}=8.0 \mathrm{~Hz}, J_{2}=7.0 \mathrm{~Hz}, J_{3}=1.0 \mathrm{~Hz}\right), 7.40(1 \mathrm{H}, J=8.0 \mathrm{~Hz}), 7.93(1 \mathrm{H}, \mathrm{s})$, $8.53(1 \mathrm{H}, \mathrm{d}, J=8.0 \mathrm{~Hz}) ;{ }^{13} \mathrm{C}$ NMR $\left(100 \mathrm{MHz}, \mathrm{DMSO}-d_{6}\right): 32.9\left(\mathrm{CH}_{3}\right), 109.8,112.8,114.6,116.8,120.3$, $121.9,123.2,129.3,132.9,157.6(\mathrm{CH}), 112.0,121.7,126.7,136.4,139.3,149.0,159.3,162.3(\mathrm{C})$.

\subsubsection{4-[2-Amino-4-(1H-indol-3-yl)pyrimidin-5-yl]benzamide (5)}

Purification of the residue by flash chromatography (cyclohexane/EtOAc 4:6) provided 5 (160 mg, 49\%) as a yellow powder.

$\mathrm{Mp}>250{ }^{\circ} \mathrm{C}$; HRMS (ESI+) calcd for $\mathrm{C}_{19} \mathrm{H}_{16} \mathrm{~N}_{5} \mathrm{O}(\mathrm{M}+\mathrm{H})^{+}$330.1355, found 330.1344; IR (KBr): 3600 $3100,1665,1610 \mathrm{~cm}^{-1}$; ${ }^{1} \mathrm{H}$ NMR $\left(400 \mathrm{MHz}, \mathrm{DMSO}-d_{6}\right): 6.62-6.68\left(2 \mathrm{H}, \mathrm{br} \mathrm{s}, \mathrm{NH}_{2}\right), 6.67(1 \mathrm{H}, \mathrm{d}$, $J=3.0 \mathrm{~Hz}), 7.05\left(1 \mathrm{H}, \mathrm{ddd}, J_{1}=8.0 \mathrm{~Hz}, J_{2}=7.0 \mathrm{~Hz}, J_{3}=1.0 \mathrm{~Hz}\right), 7.12\left(1 \mathrm{H}, \mathrm{ddd}, J_{1}=8.0 \mathrm{~Hz}, J_{2}=7.0 \mathrm{~Hz}\right.$, $\left.J_{3}=1.5 \mathrm{~Hz}\right), 7.33-7.40(4 \mathrm{H}, \mathrm{m}), 7.89(2 \mathrm{H}, \mathrm{d}, J=8.5 \mathrm{~Hz}), 7.98-8.02(1 \mathrm{H}$, br s, NH), $8.05(1 \mathrm{H}, \mathrm{s}), 8.37$ $(1 \mathrm{H}, \mathrm{d}, J=8.0 \mathrm{~Hz}), 11.26-11.32\left(1 \mathrm{H}\right.$, br s, NH); ${ }^{13} \mathrm{C}$ NMR $\left(100 \mathrm{MHz}, \mathrm{DMSO}-d_{6}\right): 111.5,120.1,121.9$, 122.6, $127.9(2 \mathrm{C}), 128.9,129.2(2 \mathrm{C}), 158.0(\mathrm{CH}), 112.7,120.4,126.1,132.6,135.9,142.0,159.9,162.6$ (C), $167.5(\mathrm{C}=\mathrm{O})$.

\subsubsection{4-[2-Amino-4-(1-methyl-1H-indol-3-yl)pyrimidin-5-yl]benzamide (6)}

Purification of the residue by flash chromatography (cyclohexane/EtOAc 4:6) provided 6 (184 mg, 54\%) as a yellow powder.

$\mathrm{Mp}>250{ }^{\circ} \mathrm{C}$; HRMS (ESI+) calcd for $\mathrm{C}_{20} \mathrm{H}_{18} \mathrm{~N}_{5} \mathrm{O}(\mathrm{M}+\mathrm{H})^{+}$344.1511, found 344.1494; IR (KBr): 3600 $3100,1681,1594 \mathrm{~cm}^{-1}$; ${ }^{1} \mathrm{H}$ NMR $\left(400 \mathrm{MHz}, \mathrm{DMSO}-d_{6}\right): 3.62\left(3 \mathrm{H}, \mathrm{s}, \mathrm{CH}_{3}\right), 6.62-6.70\left(2 \mathrm{H}, \mathrm{br} \mathrm{s}, \mathrm{NH}_{2}\right)$, $6.80(1 \mathrm{H}, \mathrm{s}), 7.06(1 \mathrm{H}, \mathrm{t}, J=7.5 \mathrm{~Hz}), 7.18(1 \mathrm{H}, \mathrm{t}, J=7.5 \mathrm{~Hz}), 7.36(2 \mathrm{H}, \mathrm{d}, J=8.0 \mathrm{~Hz}), 7.35-7.41(1 \mathrm{H}, \mathrm{br}$ s, NH), $7.41(1 \mathrm{H}, \mathrm{d}, J=8.0 \mathrm{~Hz}), 7.87(2 \mathrm{H}, \mathrm{d}, J=8.0 \mathrm{~Hz}), 7.97-8.02(1 \mathrm{H}$, br s, NH), $8.07(1 \mathrm{H}, \mathrm{s}), 8.20$ $(1 \mathrm{H}, \mathrm{d}, J=8.0 \mathrm{~Hz}) ;{ }^{13} \mathrm{C}$ NMR $\left(100 \mathrm{MHz}, \mathrm{DMSO}-d_{6}\right): 32.8\left(\mathrm{CH}_{3}\right), 109.9,120.2,121.9,122.5,127.9(2 \mathrm{C})$, $129.0(2 \mathrm{C}), 132.6,158.4(\mathrm{CH}), 112.0,120.3,126.3,132.5,136.5,141.7,159.4,162.6(\mathrm{C}), 167.5(\mathrm{C}=\mathrm{O})$.

\subsubsection{4-[2-Amino-4-(1-methyl-1H-indol-3-yl)pyrimidin-5-yl]benzoic acid (7)}

Purification of the residue by flash chromatography (EtOAc/MeOH 9:1) provided 7 (207 mg, 60\%) as a yellow powder.

$\mathrm{Mp}>250{ }^{\circ} \mathrm{C}$; HRMS (ESI+) calcd for $\mathrm{C}_{20} \mathrm{H}_{17} \mathrm{~N}_{4} \mathrm{O}_{2}(\mathrm{M}+\mathrm{H})^{+}$345.1352, found 345.1342; IR (KBr): $3250-2800,1608 \mathrm{~cm}^{-1}$; ${ }^{1} \mathrm{H}$ NMR $\left(400 \mathrm{MHz}, \mathrm{DMSO}-d_{6}\right): 3.63\left(3 \mathrm{H}, \mathrm{s}, \mathrm{CH}_{3}\right), 6.66-6.72\left(2 \mathrm{H}, \mathrm{br} \mathrm{s}, \mathrm{NH}_{2}\right)$, $6.83(1 \mathrm{H}, \mathrm{s}), 7.05\left(1 \mathrm{H}, \mathrm{ddd}, J_{1}=8.0 \mathrm{~Hz}, J_{2}=7.0 \mathrm{~Hz}, J_{3}=1.0 \mathrm{~Hz}\right), 7.17\left(1 \mathrm{H}\right.$, ddd, $J_{1}=8.0 \mathrm{~Hz}, J_{2}=7.0 \mathrm{~Hz}$, $\left.J_{3}=1.0 \mathrm{~Hz}\right), 7.38-7.42(3 \mathrm{H}, \mathrm{m}), 7.91(2 \mathrm{H}, \mathrm{d}, J=8.5 \mathrm{~Hz}), 8.08(1 \mathrm{H}, \mathrm{s}), 8.16(1 \mathrm{H}, \mathrm{d}, J=8.0 \mathrm{~Hz})$, 12.73-13.09 (1H, br s, COOH); ${ }^{13} \mathrm{C}$ NMR (100 MHz, DMSO- $\left.d_{6}\right): 32.8\left(\mathrm{CH}_{3}\right), 109.9,120.2,121.9,122.4$, 129.3 (2C), 129.7 (2C), 132.7, $158.4(\mathrm{CH}), 111.9,120.1,126.3,129.1,136.5,143.3,159.4,162.6(\mathrm{C})$, $167.2(\mathrm{C}=\mathrm{O})$.

\subsubsection{4-(1-Methyl-1H-indol-3-yl)-5,5'-bipyrimidin-2-amine (8)}

Purification of the residue by flash chromatography (cyclohexane/EtOAc 2:8) provided 8 (95 mg, 31\%) as a yellow powder. 
Mp $>250{ }^{\circ} \mathrm{C}$; HRMS (ESI+) calcd for $\mathrm{C}_{17} \mathrm{H}_{15} \mathrm{~N}_{6}(\mathrm{M}+\mathrm{H})^{+}$303.1358, found 303.1344; IR (KBr): 3400-3100, $1638 \mathrm{~cm}^{-1}$; ${ }^{1} \mathrm{H}$ NMR (400 MHz, DMSO-d $)$ : $3.69\left(3 \mathrm{H}, \mathrm{s}, \mathrm{CH}_{3}\right), 6.80-6.87\left(2 \mathrm{H}\right.$, br s, $\left.\mathrm{NH}_{2}\right)$, $7.04(1 \mathrm{H}, \mathrm{s}), 7.05(1 \mathrm{H}, \mathrm{t}, J=7.5 \mathrm{~Hz}), 7.18(1 \mathrm{H}, \mathrm{t}, J=7.5 \mathrm{~Hz}), 7.43(1 \mathrm{H}, \mathrm{d}, J=8.0 \mathrm{~Hz}), 7.99(1 \mathrm{H}, \mathrm{d}$, $J=8.0 \mathrm{~Hz}), 8.21(1 \mathrm{H}, \mathrm{s}),, 8.70(2 \mathrm{H}, \mathrm{s}), 9.09(1 \mathrm{H}, \mathrm{s}) ;{ }^{13} \mathrm{C}$ NMR $\left(100 \mathrm{MHz}, \mathrm{DMSO}-d_{6}\right): 32.8\left(\mathrm{CH}_{3}\right), 110.1$, $120.3,122.0,122.1,132.7,156.5(3 \mathrm{C}), 159.1(\mathrm{CH}), 111.7,114.2,125.9,132.5,136.6,160.1,163.2(\mathrm{C})$.

\subsubsection{4-(1-Methyl-1H-indol-3-yl)-5-(pyridin-4-yl)pyrimidin-2-amine (9)}

Purification of the residue by flash chromatography (cyclohexane/EtOAc 4:6) provided 9 (98 mg, 33\%) as a yellow powder.

$\mathrm{Mp}>250{ }^{\circ} \mathrm{C}$; HRMS (ESI+) calcd for $\mathrm{C}_{18} \mathrm{H}_{16} \mathrm{~N}_{5}(\mathrm{M}+\mathrm{H})^{+}$302.1406, found 302.1393; IR (KBr): 3350-3100, $1649 \mathrm{~cm}^{-1}$; ${ }^{1} \mathrm{H}$ NMR (400 MHz, DMSO-d $)$ : $3.67\left(3 \mathrm{H}, \mathrm{s}, \mathrm{CH}_{3}\right), 6.76-6.83\left(2 \mathrm{H}\right.$, br s, $\left.\mathrm{NH}_{2}\right)$, $7.01(1 \mathrm{H}, \mathrm{s}), 7.04\left(1 \mathrm{H}, \mathrm{ddd}, J_{1}=8.0 \mathrm{~Hz}, J_{2}=7.0 \mathrm{~Hz}, J_{3}=1.0 \mathrm{~Hz}\right), 7.18\left(1 \mathrm{H}, \mathrm{ddd}, J_{1}=8.0 \mathrm{~Hz}, J_{2}=7.0 \mathrm{~Hz}\right.$, $\left.J_{3}=1.0 \mathrm{~Hz}\right), 7.30(2 \mathrm{H}, \mathrm{m}), 7.42(1 \mathrm{H}, \mathrm{d}, J=8.0 \mathrm{~Hz}), 8.02(1 \mathrm{H}, \mathrm{d}, J=8.0 \mathrm{~Hz}), 8.14(1 \mathrm{H}, \mathrm{s}), 8.49(2 \mathrm{H}, \mathrm{d}$, $J=5.5 \mathrm{~Hz}) ;{ }^{13} \mathrm{C}$ NMR $\left(100 \mathrm{MHz}, \mathrm{DMSO}-d_{6}\right): 32.8\left(\mathrm{CH}_{3}\right), 110.1,120.3,122.0,122.1,124.0(2 \mathrm{C}), 132.6$, $149.8(2 \mathrm{C}), 158.6(\mathrm{CH}), 111.8,118.5,126.1,136.6,146.5,159.5,162.9(\mathrm{C})$.

\subsubsection{5-Iodo-4-(1-Methyl-1H-indol-3-yl)pyrimidin-2-amine (10)}

Iodine $(1.13 \mathrm{~g}, 4.46 \mathrm{mmol})$ was added to the solution of compound $\mathbf{I}(1.0 \mathrm{~g}, 4.46 \mathrm{mmol})$ in DMSO $(50 \mathrm{ml})$. The mixture was stirred overnight at $25^{\circ} \mathrm{C}$. The reaction was quenched with a saturated aqueous solution of sodium thiosulfate and was extracted with EtOAc. The organic phase was washed with a brine solution and then dried over $\mathrm{MgSO}_{4}$, filtrated and evaporated onto silica gel. Purification by flash chromatography (cyclohexane/ethyl acetate 1:9) provided $\mathbf{1 0}(1.20 \mathrm{~g}, 3.43 \mathrm{mmol}, 77 \%$ ) as a yellow powder.

$\mathrm{Mp}=185-188^{\circ} \mathrm{C}$; HRMS (ESI+) calcd for $\mathrm{C}_{13} \mathrm{H}_{12} \mathrm{IN}_{4}(\mathrm{M}+\mathrm{H})^{+}$351.0107, found 351.0091; IR $(\mathrm{KBr})$ : 3650-3200, $1633 \mathrm{~cm}^{-1} ;{ }^{1} \mathrm{H}$ NMR (400 MHz, DMSO-d $): 3.89\left(3 \mathrm{H}, \mathrm{s}, \mathrm{CH}_{3}\right), 6.67-6.77\left(2 \mathrm{H}\right.$, br s, $\left.\mathrm{NH}_{2}\right)$, $7.16\left(1 \mathrm{H}, \mathrm{ddd}, J_{1}=8.0 \mathrm{~Hz}, J_{2}=7.0 \mathrm{~Hz}, J_{3}=1.0 \mathrm{~Hz}\right), 7.25\left(1 \mathrm{H}, \quad \mathrm{ddd}, J_{1}=8.0 \mathrm{~Hz}, J_{2}=7.0 \mathrm{~Hz}\right.$, $\left.J_{3}=1.0 \mathrm{~Hz}\right), 7.51\left(1 \mathrm{H}, \mathrm{dt}, J_{1}=8.0 \mathrm{~Hz}, J_{2}=1.0 \mathrm{~Hz}\right), 8.32\left(1 \mathrm{H}, \mathrm{ddd}, J_{1}=8.0 \mathrm{~Hz}, J_{2}=1.0 \mathrm{~Hz}, J_{3}=0.5 \mathrm{~Hz}\right)$, $8.43(1 \mathrm{H}, \mathrm{s}), 8.49(1 \mathrm{H}, \mathrm{s}) ;{ }^{13} \mathrm{C}$ NMR $\left(100 \mathrm{MHz}, \mathrm{DMSO}-d_{6}\right): 33.0\left(\mathrm{CH}_{3}\right), 110.1,120.5,122.1,122.7$, $133.2,165.8(\mathrm{CH}), 74.7,112.8,126.6,136.5,161.9,162.1(\mathrm{C})$.

\section{Acknowledgments}

The authors thank the European Union Prokinase Research Consortium for financial support and Bertrand Légeret for mass spectrometry analysis. This research was also supported by grants from "Association France-Alzheimer Finistère" (LM), CRITT Santé Bretagne (LM) and "Fondation Jérôme Lejeune" (LM).

\section{References and notes}

1 L.H. Franco, E. Bal de Kier Joffé, L. Puricelli, M. Tatian, A.M. Seldes and J.A. Palermo, J. Nat. Prod. 61 (1998), p. 1130.

2 L.H. Franco and J.A. Palermo, Chem. Pharm. Bull. 51 (2003), p. 975.

3 M. Gompel, M. Leost, E. Bal de Kier Joffé, L. Puricelli, L.H. Franco, J. Palermo and L. Meijer, Bioorg. Med. Chem. Lett. 14 (2004), p. 1703. 4 M.A.A. Radwan and M. El-Sherbiny, Bioorg. Med. Chem. 14 (2007), p. 1206.

5 K. Bettayeb, O.M. Tirado, S. Marionneau-Lambot, Y. Ferandin, O. Lozach, J.C. Morris, S. Mateo-Lozano, P. Drueckes, C. Schächtele, M.H.G. Kubbutat, F. Liger, B. Marquet, B. Joseph, A. Echalier, J.A. Endicott, V. Notario and L. Meijer, Cancer Res. 67 (2007), p. 8325.

6 A. Echalier, K. Bettayeb, Y. Ferandin, O. Lozach, M. Clément, A. Valette, F. Liger, B. Marquet, J.C. Morris, J.A. Endicott, B. Joseph and L. Meijer, J. Med. Chem. 51 (2008), p. 737.

7 E. Rossignol, A. Youssef, P. Moreau, M. Prudhomme and F. Anizon, Tetrahedron 63 (2007), p. 10169.

8 E. Rossignol, E. Debiton, D. Fabbro, P. Moreau, M. Prudhomme and F. Anizon, Anti-Cancer Drugs 19 (2008), p. 789.

9 M.V. Jovanovic, Heterocycles 22 (1984), p. 1195.

10 J. O’Brien, I. Wilson, T. Orton and F. Pognan, Eur. J. Biochem. 267 (2000), p. 5421.

11 R. Kimura, K. Kamino, M. Yamamoto, A. Nuripa, T. Kida, H. Kazui, R. Hashimoto, T. Tanaka, T. Kudo, H. Yamagata, Y. Tabara, T. Miki, H. Akatsu, K. Kosaka, E. Funakoshi, K. Nishitomi, G. Sakaguchi, A. Kato, H. Hattori, T. Uema and M. Takeda, Hum. Mol. Genet. 16 (2007), p. 15.

12 I. Branchi, Z. Bichler, L. Minghetti, J.M. Delabar, F. Malchiodi-Albedi, M.C. Gonzalez, Z. Chettouh, A. Nicolini, C. Chabert, D.J. Smith, E.M. Rubin, D. Migliore-Samour and E. Alleva, J. Neuropathol. Exp. Neurol. 63 (2004), p. 429.

13 S. Leclerc, M. Garnier, R. Hoessel, D. Marko, J.A. Bibb, G.L. Snyder, P. Greengard, J. Biernat, E.-M. Mandelkow, G. Eisenbrand and L. Meijer, J. Biol. Chem. 276 (2001), p. 251. 
14 S. Bach, M. Knockaert, O. Lozach, J. Reinhardt, B. Baratte, S. Schmitt, S.P. Coburn, L. Tang, T. Jiang, D.C. Liang, H. Galons, J.F. Dierick, F. Totzke, C. Schächtele, A.S. Lerman, A. Carnero, Y. Wan, N. Gray and L. Meijer, J. Biol. Chem. 280 (2005), p. 31208.

15 A. Primot, B. Baratte, M. Gompel, A. Borgne, S. Liabeuf, J.L. Romette, F. Costantini and L. Meijer, Protein Exp. Purif. 20 (2000), p. 394.

16 J. Reinhardt, Y. Ferandin and L. Meijer, Protein Exp. Purif. 54 (2007), p. 101. 